\title{
Análisis del foro online de un máster interuniversitario por aula remota en clave de emociones
}

\section{Online forum analysis of a joint master's degree by remote classes in terms of emotions}

\author{
Miguel Romero Gutiérrez \\ Universidad de Almería. Almería, España \\ miguelromero@ual.es \\ María Martínez Chico \\ Universidad de Almería. Almería, España \\ maria.martinez.chico@gmail.com \\ María Rut Jiménez Liso \\ Universidad de Almería. Almería, España \\ mrjimene@ual.es
}

\begin{abstract}
Resumen
Con intención de detectar las emociones expresadas en una asignatura desarrollada en un programa de educación superior a distancia (Máster Interuniversitario de Educación Ambiental), se ha realizado el análisis de un foro online, espacio idóneo de reflexión en el que se fomenta el aprendizaje colaborativo, lo que aporta información del efecto de la asignatura y su significatividad para los estudiantes. Lo distintivo de esta docencia virtual desarrollada en la asignatura es que está centrada en promover y hacerles vivir enfoques de enseñanza basados en la indagación entre futuros educadores ambientales. El aspecto emocional es determinante en la educación ambiental, por ello, a través de una metodología cualitativa, hemos abordado el análisis de las aportaciones de los estudiantes al foro online que nos ha permitido identificar emociones expresadas, sus detonantes y las actividades que pueden funcionar como estímulo en su futuro como educadores-as ambientales. La implicación emocional destacada en sus respuestas muestra que la asignatura no les deja indiferentes, destacando la satisfacción experimentada y su preocupación ante situaciones con posibles consecuencias negativas.
\end{abstract}

Palabras clave

Foros online, Formación de Educadores, Educación Ambiental, Emociones, Enseñanza por indagación.

\begin{abstract}
Intended to evaluate the efficiency of a subject developed in a remote online higher education program (Interuniversity Environmental Education Joint Master Degree), we have developed an analysis of an online forum, an excellent place to reflect where the collaborative learning is fostered, providing information on the effect of the subject and its significance for students. What is characteristic of the virtual teaching developed in the subject is that is focused on promoting and experiencing inquiry-based teaching approaches among future environmental educators. The emotional aspect proves crucial for environmental education thus, through a qualitative methodology, we had addressed the analysis of the students' contributions in the online forum what let us identify emotions expressed, its triggers and the activities that can work as an incentive in their future as environmental educators. The highlighted emotional involvement expressed in theirs posts shows that the subject doesn't remain indifferent, emphasizing the satisfaction experimented and theirs worry about situations with possible negative consequences.
\end{abstract}

Key words

Online Forums, Teachers' Education, Environmental Education, Emotions, Inquiry based Learning. 
In memoriam: Este artículo nunca hubiera sido possible sin el apoyo desinteresado, los comentarios, las enseñanzas sobre actitudes-flow y la amistad del profesor Francisco Gil

Cuadra. Sirvan estas palabras para expresar nuestro infinito agradecimiento por tanto.

\section{Educación superior y tecnologías para el aprendizaje colectivo virtual}

Actualmente nos encontramos en una realidad tecnologizada cuya repercusión en las prácticas de aula implica un mayor uso de los recursos digitales y cambios de organización de las clases que promuevan el trabajo colaborativo en la construcción del conocimiento (Area \& Sanabria, 2014)

La educación superior española se está viendo sometida a dos importantes condiciones que afectan a su modelo educativo. Por una parte, la reorganización del sistema de enseñanza al marco del Espacio Europeo de Educación Superior (EEES) para adaptarlo a un modelo de formación centrado en el trabajo y el aprendizaje de los estudiantes, y, por otra parte, la expansión cada vez mayor de los recursos informáticos y del uso de Internet dentro del trabajo académico universitario (de la Garza Aguilar, 2013). Conscientes de este hecho, es necesario dar respuesta a la demanda de competencias digitales y comunicativas, necesarias para desenvolverse en el actual mundo tecnologizado (Gutiérrez-Martín, Palacios-Picos, \& Torrego-Egido, 2010; Zapata-Ros, 2011), es decir, el uso crítico de la TIC, para recuperar, evaluar, almacenar, producir, presentar, intercambiar información, etc. Para lograr estas competencias (básicas en muchas titulaciones), las universidades han incorporado en sus ambientes de aprendizaje distintas herramientas tecnológicas (wikis, herramientas colaborativas online, blogs, foros, redes y marcadores sociales) para el uso y beneficio tanto del alumnado como de los equipos docentes (Salgado Santamaría, González Conde, \& Zamarra López, 2013). Esta incorporación no significa, necesariamente, una mejora de las prácticas educativas (Onrubia, 2005), pero permite a sus usuarios interactuar y colaborar entre sí creando contenidos y generando comunidades virtuales de aprendizaje transformando los entornos tradicionales de enseñanzas en entornos en los que se lleven a cabo metodologías caracterizadas por la virtualización del proceso de enseñanza (Díez Gutiérrez, 2012).

Las dos condiciones anteriores, centrar la enseñanza en el trabajo del alumno (EEES) y el uso de TIC, nos hacen tener presente la adecuación y optimización a las TIC de los programas de títulos y de asignaturas. Ello dependerá en gran parte de cómo las instituciones, en nuestro caso, las universidades, incorporen estos elementos en los procesos de enseñanza-aprendizaje, ya que esto determinará el impacto de las innovaciones realizadas (Boza Carreño \& Conde, 2015) y permitirá trazar un plan que resulte útil para priorizar tareas y modificaciones en el diseño, así como revisar el papel del docente y del estudiante en cada aula, observando cuándo y por qué se participa (Rubio Serrano, Puig Rovira, Martín García, \& Palos Rodríguez, 2015).

Esto se hace aún más necesario, tal y como describen Fuentes Agustí y Guillamón Ramos (2006), en aquellas asignaturas impartidas íntegramente online que tienen como mayor reto implicar e involucrar a los estudiantes en el desarrollo de la asignatura, de manera que su participación activa constituya, en sí misma, un mecanismo de aprendizaje, formando parte de la propia metodología de la asignatura (Ching \& Hsu, 2011).

Este es el caso del Máster Interuniversitario de Educación Ambiental pues, como desarrollaremos a continuación, este máster tiene la particularidad que los estudiantes asisten a las clases simultáneamente (en sincronía) en siete sedes a través de aula remota, lo que genera una mezcla entre docencia a distancia y docencia in situ y supone

Análisis del foro online de un máster interuniversitario por aula remota en clave de emociones. Miguel Romero Gutiérrez, María Martínez Chico y M. Rut Jiménez Liso.

Página 2 de 21 
un reto para el profesorado de fomentar la participación de manera similar, tanto del alumnado presente en la sede donde se retransmite la docencia, como aquel que asiste por aula remota. A esto hemos de sumar el desafío de lograr que el máster en cuestión genere la ambientalización del currículo, como se viene demandando para los programas de formación de educadores y docentes en educación superior (Ryan y Tilbury, 2013) y se incentive la participación en la acción ciudadana (Marques \& Reis, 2017).

Los mencionados cambios metodológicos en la educación superior deben suponer cambios en el planteamiento de la evaluación, más concretamente en aquellos programas de educación virtual (Baxter \& Haycock, 2014), donde sus características hacen imprescindible ampliar la evaluación al proceso formativo colectivo, integrando todas las variables implicadas en el proceso de enseñanza-aprendizaje (Sarramona i López, 2001), pues la no presencialidad o la distancia entre los estudiantes obliga a generar espacios para el aprendizaje colectivo que mantengan un propósito de evaluación formativa.

\section{Foros online: Una oportunidad para el aprendizaje y la evaluación colaborativos}

Los foros virtuales, dada su función comunicativa e interactiva, ofrecen un espacio idóneo para favorecer el aprendizaje compartido y permitir una actividad docente que promueva la evaluación formativa.

Estas herramientas tecnológicas resultan especialmente útiles en asignaturas no presenciales, donde la lejanía entre estudiantes y docentes supone un reto tanto para la entrega de documentos como para el intercambio de mensajes, así como un riesgo para el abandono y/o falta de participación en la asignatura (Martínez-Villar, Gutiérrez Pérez, \& Perales-Palacios, 2016). De esta forma, el foro online se convierte en una "social network" para empoderarse y adquirir competencias a través de la construcción general del conocimiento por medio de una metodología colaborativa de discusión en red (Mokoena, 2013).

Gracias al entorno de diálogo entre iguales, el contenido de los foros puede ser utilizado en el proceso de análisis de la optimización de los programas formativos diseñados, superando las evaluaciones de programas e-learning, de los que suelen surgir un enfoque parcial de evaluación, centrados en la actividad formativa, los materiales de formación, las plataformas tecnológicas o la relación coste/beneficio; teniendo como finalidad alguno de estos aspectos: comprobar el nivel de cumplimiento de los objetivos educativos, mejorar la propia acción formativa y determinar el retorno de la inversión realizada (Rubio, 2003).

Además, los foros online ofrecen una plataforma para el aprendizaje colaborativo, como se ha podido comprobar desde los inicios de las prácticas educativas formales en contextos virtuales (CSCL, BSCW, Aulas Virtuales, etc.). A pesar de esta presencia, como destaca Barberá (2006), la evaluación colaborativa, incluso estando asociada a todas las plataformas de e-learning (foros, debates, hilos de conversación, etc.) apenas se ha tratado, siendo un campo por explorar, quizá debido a que no se sabe muy bien cómo extraer indicadores para la evaluación del aprendizaje. Una de las principales ventajas pedagógicas que se desprenden de una acción colaborativa en un contexto virtual respecto a las prácticas presenciales, es la posibilidad de evaluar tanto el producto colaborativo como también el proceso, que nos aporta la tecnología (Gregori \& Elena, 2006).

Análisis del foro online de un máster interuniversitario por aula remota en clave de emociones. Miguel Romero Gutiérrez, María Martínez Chico y M. Rut Jiménez Liso. 
Al considerar la evaluación de la eficiencia (Álvarez-Pinilla, 2014) de este tipo de programas de formación como el que nos ocupa, debemos considerar los tres elementos que, según Onrubia (2005), interactúan en el proceso de enseñanza-aprendizaje en entornos virtuales: La actividad mental constructivista del alumno que aprende, la ayuda sostenida y continuada del que enseña y el contenido que es objeto de enseñanza y aprendizaje. La interacción de estos tres elementos junto a las herramientas tecnológicas, los materiales, las actividades y la interactividad se debe cuidar con el fin de estimular la participación libre y plural, incentivar el diálogo y articular múltiples informaciones y conexiones, creando una red entre docentes y discentes de intercambios formada por contenidos curriculares e instrumentos pedagógicos y que promuevan la materialización de la acción comunicativa potenciadora del proceso de enseñanzaaprendizaje (Onrubia, 2005; Silva, 2005).

En este sentido hemos encontrado antecedentes que utilizaban los foros online para evaluar logros académicos y satisfacción (Ehuletche, De Stefano, Banno, \& González, 2007), para analizar las interacciones (Tió Torriente, Estrada Sentí, González Hernández, \& Rodríguez Ortega, 2011), el papel del diálogo que surge en dicho entorno, para buscar patrones de usuarios tipo (Noriega Borge, 2012), para construir el concepto de aula como comunidad de aprendizaje, para detectar en qué momentos del proceso de enseñanza-aprendizaje utilizaban conocimientos didácticos del contenido (Jiménez-Liso y López-Gay, 2012) o para analizar contenidos didácticos presentes en los mensajes del alumnado (Martínez-Villar et al., 2016).

El foro online objeto de este trabajo se enmarca en una asignatura de un máster interuniversitario de educadores ambientales, cuyo efecto debe ser evaluado desde la perspectiva emocional, además de las habituales perspectivas cognitivas: contenidos y procesos (Romero-Gutiérrez, Jiménez-Liso, \& Martínez-Chico, s. f., en revisión). En el presente artículo abordamos este aspecto, cuya consideración continúa ganando peso en la investigación educativa: Las emociones.

\section{Las emociones en el aprendizaje online}

Hoy en día, las emociones han adquirido una gran importancia en nuestra sociedad; prueba de ello sirve la popularidad que se ha otorgado a diferentes autores que han escrito obras de gran éxito (Goleman, 1996; Mora Teruel, 2013; Punset, 2009). De igual modo, se ha comenzado a prestar mayor atención a las emociones en la investigación educativa, en el proceso de enseñanza/aprendizaje, en la vida de los docentes en general y de los de ciencias, en particular (Borrachero Cortés, Bermejo García, \& Costillo Borrego, 2014; Dávila Acedo, Borrachero Cortés, Cañada Cañada, Martínez Borreguero, \& Sánchez Martín, 2015; Feidakis, Daradoumis, Caballé, \& Conesa, 2014). Internet funciona como una oportunidad un tanto particular para la expresión de la multiplicidad del self, lo cual pone en entredicho la asociación entre el establecimiento de las relaciones online y la "preocupante" tendencia a volvernos progresivamente seres asociales, aislados y depresivos. En este sentido, las emociones no han de ser analizadas en función del impacto que tienen sobre el aprendizaje, sino en cuanto a dimensiones esenciales de este mismo aprendizaje, ya que la calidad emergente de la emoción tiene implicaciones importantes para la educación (Aires, Teixeira, Azecedo, Gaspar, \& Silva, 2006).

La relación emocional con nuevas herramientas y contenidos de aprendizaje supone una línea de estudio, especialmente interesante en relación con el e-learning y la formación a distancia, en la que cada vez más investigaciones educativas se centran en el diseño y

Análisis del foro online de un máster interuniversitario por aula remota en clave de emociones. Miguel Romero Gutiérrez, María Martínez Chico y M. Rut Jiménez Liso. 
evaluación de entornos de aprendizaje online que incorporan recursos, estrategias y actividades relacionadas con la regulación emocional, y en los que se analiza la comunicación emocional en el aprendizaje online (Aires et al., 2006; Carmi, Arnon, \& Orion, 2015; Rebollo Catalán, García Pérez, Barragán Sánchez, Buzón García, \& Vega Caro, 2008).

El estudio emocional en relación con el uso de las tecnologías como recurso de aprendizaje resulta especialmente necesario, dados los elevados índices de abandono y fracaso que presentan los modelos de formación a distancia (Aires et al., 2006; Rebollo Catalán et al., 2008). Las emociones vivenciadas en el contexto de las comunidades virtuales de aprendizaje pueden ser estudiadas en el ámbito del análisis de las declaraciones producidas en los foros de discusión (Aires et al., 2006), donde los mensajes publicados y discutidos por estudiantes y docentes son analizados mediante metodologías cualitativas.

Siendo muy amplio el abanico de posibilidades para identificar o reconocer las emociones experimentadas por los participantes en los cursos de formación: preguntas abiertas o cerradas, entrevistas, encuestas, cuestionarios, etc. (Chawla \& Cushing, 2007), en nuestro caso, al tratarse de un título a distancia, hemos descartado las opciones que requiriesen presencialidad, tratando de facilitar un ambiente de reflexión y libertad, en el que los alumnos no se sintiesen juzgados por un responsable (Hyatt, 2005). De este modo, el foro online sirve además como una actividad más del curso, con la que se realizase una auto-reflexión crítica sobre el mismo del cual pudiesen surgir nuevas cuestiones a tratar (Lillis \& Turner, 2001).

\section{Educación ambiental integral: Indagación y emociones}

Para que la educación ambiental pueda convertirse en el proceso que genere el germen de una nueva cultura y de nuevas formas de pensamiento, necesita hacer sentir un compromiso por mejorar la situación del medio y la interacción humana a través de la educación, el conocimiento y las habilidades. Estas herramientas deben ayudar a conocer sus límites y posibilidades y generar en las personas la posibilidad de apasionarse por el mundo, de comprometerse a pensar y generar respuestas más adecuadas a los problemas que se plantean los grupos humanos, lo que significa aprender a pensar. En este sentido, como plantean Romero Cuevas (2006), la educación ha de abordarse con la intención de conseguir transformaciones profundas, convertirse en instancia formadora de nuevas formas de "saber, hacer, sentir, tener, estar..." y plantear a los humanos el reto de "crecer y soñar" para ser integralmente humanos.

Desde su legitimación como ámbito pedagógico, la educación ambiental se ha encontrado en un permanente disenso en cuanto a enfoques y posturas metodológicas adoptadas (Poza Vilches et al., 2016). Al margen de la concepción clásica del aprendizaje como resultado de una transmisión de saberes, la educación ambiental pone en evidencia los lazos estrechos entre saber y acción que se construyen recíprocamente. Sauvé (2010) destaca la idea de que el saber no es necesariamente previo a la acción, que él la atraviesa al tomar forma, y que, al transformarse la acción, se alimenta del saber, lo confronta, lo valida. Acción y reflexión no se entienden ya como etapas separadas, sino que ser y actuar son indisociables en nuestra dinámica de vida. Es así como podemos aprender de nuestra interrelación con el mundo: sensibilidad, sensorialidad, intuición, racionalidad, experiencia empírica, sea de naturaleza cinética, comunicacional, artística, científica, tecnológica, etc. Entendemos que ese es el caso de todas las formas de aprendizaje, en concreto del aprendizaje de las ciencias, con la

Análisis del foro online de un máster interuniversitario por aula remota en clave de emociones. Miguel Romero Gutiérrez, María Martínez Chico y M. Rut Jiménez Liso. 
particularidad de que en materia de medio ambiente el carácter social, colectivo y resolutivo de la acción está más marcado.

De la integración de la educación científica y ambiental, para afrontar los actuales desafíos de la humanidad que requieren la toma de decisiones sobre aspectos que afectan a la salud, calidad de vida, o al uso de los recursos materiales y energéticos..., y que en definitiva conllevan unas repercusiones medioambientales y económicas, se plantea la alfabetización científica como objetivo internacional clave (Organization for Economic Co-operation and Development [OCDE], 2016).

Estas metas educativas requieren una reflexión acerca de qué tipo de enseñanza favorece la formación de ciudadanos científicamente alfabetizados, a la par que contempla los indisociables saber y acción de la educación científica y ambiental (Reis \& Marques, 2016). Esto conlleva identificar qué enfoque metodológico y qué evaluación adoptar para favorecer dichos resultados de aprendizaje. En este sentido, la enseñanza de las ciencias por indagación presenta una complementariedad importante con la educación ambiental en el sentido de que permite abordar preguntas o problemas en relación con cuestiones socio-científicas de repercusión ambiental y construir respuestas argumentadas, fundamentadas en un razonamiento que esté apoyado en pruebas (National Research Council [NRC], 2012). De manera que, al partir de una "pregunta que enganche" a los estudiantes y no les deje indiferentes, relacionada con problemas ambientales (agua, eficiencia energética, residuos y transformación de sustancias, etc.), se estaría conectando conocimiento racional y emocional, nuestra identificación y vinculación con la Tierra, algo que según Chawla (2006) resulta esencial en educación ambiental. Recordemos que uno de los objetivos que persigue la educación ambiental es vivir y desenvolverse en el mundo con una inteligencia comprensiva que combine una identificación profunda de la naturaleza con la comprensión de sus procesos (Cobb, 1977).

En un análisis profundo para conocer qué actividades son efectivas en la educación ambiental, Hungerford y Volk (1990) concluyeron algunas de las variables más importantes que al combinarse determinan acciones responsables efectivas: "sensibilidad medioambiental, conocimiento profundo del problema, implicación personal en el problema, locus de control, conocimiento y habilidad en el uso de las estrategias de acción medioambientales y la intención de actuar. De acuerdo con Chawla (2006), estas variables podrían cubrirse trabajando los sentimientos y las emociones (sensibilidad medioambiental, implicación personal, locus de control e intención de actuar) así como el conocimiento (conocimiento del problema, conocimiento y habilidad en el uso de estrategias de acción).

$\mathrm{Al}$ encontrarse las emociones integradas en el proceso de aprendizaje, podemos enfocar la evaluación educativa desde una perspectiva de investigación cualitativa. Sin embargo no deberemos asumir el centrarnos únicamente en los aspectos emocionales e interpretativos de la propia experiencia, sino que la capacidad de exploración crítica de este enfoque nos va a permitir unir los ámbitos emocionales y racionales del aprendizaje ambiental (Chawla, 2006).

Como afirma Gomera Martínez (2008) la educación ambiental ha de pretender ser un activador de la conciencia ambiental de los individuos, término definido como el sistema de vivencias, conocimientos y experiencias que el individuo utiliza activamente en su relación con el medio ambiente, y en el que podemos distinguir cuatro dimensiones: Cognitiva (grado de información y conocimiento sobre cuestiones relacionadas con el medio ambiente -ideas-); afectiva (percepción del medio ambiente, creencias y sentimientos en materia medioambiental -emociones-); conativa

Análisis del foro online de un máster interuniversitario por aula remota en clave de emociones. Miguel Romero Gutiérrez, María Martínez Chico y M. Rut Jiménez Liso. 
(predisposición a adoptar criterios pro-ambientales en la conducta, manifestando interés o predisposición a participar en actividades y aportar mejoras -actitudes-); y activa (realización de prácticas y comportamientos ambientalmente responsables, tanto individuales como colectivos, incluso en situaciones comprometidas o de presión conductas-).

Para que un individuo adquiera un compromiso con el desarrollo sostenible, o como más recientemente se viene hablando de "en un mundo en decrecimiento", tal que integre la variable ambiental como valor en su toma de decisiones diaria es necesario que éste alcance un grado adecuado de conciencia ambiental a partir de unos niveles mínimos en estas dimensiones. Consideramos por tanto que la formación de educadores ambientales ha de orientarse de manera que se contribuya a desarrollar sus propias dimensiones, de las cuales, en este trabajo, nos centraremos especialmente en el aspecto emocional.

Por todo lo expuesto, abordamos la formación inicial de futuros educadores y la evaluación realizada desde el tripartito Educación Ambiental - Enseñanza por Indagación - Emociones. A continuación, presentamos las preguntas y los objetivos de investigación que han guiado este trabajo.

\section{Preguntas y objetivos de investigación}

En el presente artículo, utilizamos como objeto de estudio un foro online propuesto en la asignatura Enfoque de enseñanza por indagación para educadores ambientales del Máster Interuniversitario Andaluz de Educación Ambiental (semipresencial por aula remota). En el título de la asignatura se explicita el enfoque de enseñanza de indagación como objetivo principal, con la finalidad de dar a conocer sus características a los participantes y, en palabras de sus docentes, hacer vivir la indagación como enfoque de enseñanza a través de experiencias concretas, a todos los estudiantes del máster de las siete sedes implicadas. Por ello, las docentes de la asignatura se preguntan ¿cómo podemos saber qué perciben y experimentan nuestros estudiantes al "vivir" la asignatura sobre el enfoque de enseñanza por indagación?, considerando que se trata de un máster a distancia. Una posible vía para averiguarlo es analizar el foro "diario de clase", donde los estudiantes deben narrar lo que han aprendido tras finalizar cada sesión (ver indicaciones en Figura 1).

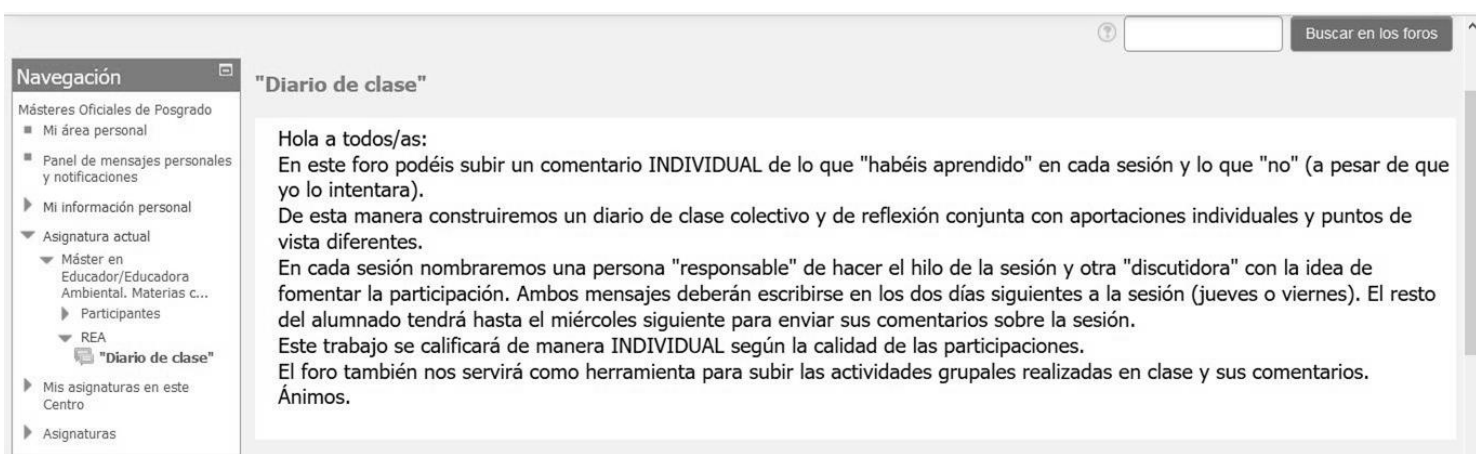

Figura 1. Captura de pantalla del foro "Diario de clase". (tomada de Romero-Gutiérrez et al., s. f., en revisión)

Por un lado, y conscientes de las dificultades que conlleva el análisis de la interacción y construcción colaborativa del conocimiento (Gros Salvat \& Silva, 2006) analizaremos el contenido (ámbitos conceptual y procedimental) que explicitan haber aprendido, es

Análisis del foro online de un máster interuniversitario por aula remota en clave de emociones. Miguel Romero Gutiérrez, María Martínez Chico y M. Rut Jiménez Liso.

Página 7 de 21 
decir, las características del enfoque de enseñanza por indagación que los estudiantes utilizan en sus mensajes al foro (Romero-Gutiérrez et al., s. f., en revisión). Por otro lado, al tratarse de narraciones libres sobre la experiencia vivida, es de esperar que esta reflexión sobre lo aprendido venga entrelazada con connotaciones emocionales (aspecto emocional), de manera que no reducimos la consideración del proceso de enseñanzaaprendizaje al aspecto meramente cognitivo, sino que consideramos también el aspecto emocional.

De esta forma, en el presente artículo, nos centraremos en conocer:

- ¿Qué emociones detectamos en los mensajes de los estudiantes al foro online y en qué momentos de la asignatura? Dado que los procesos de enseñanzaaprendizaje no son meramente cognitivos, sino que están cargados de sentimientos (Brígido Mero et al., 2010; Mellado Jiménez et al., 2014; Otero, 2006) de igual manera que identificaremos lo que han aprendido (conocimientos y procedimientos), en las narraciones de los estudiantes en el foro identificaremos qué emociones narran, para así poder conocer la conexión entre ambos aspectos: qué emociones describen (propias o ajenas) mientras viven la enseñanza por indagación.

Para abordar este propósito, en primer lugar, contextualizaremos la asignatura y el foro objeto de estudio y, a continuación, identificaremos los momentos clave en las declaraciones de los estudiantes en el foro con relación a las emociones (propias o ajenas) declaradas en sus mensajes. Para ello, definiremos bajo qué criterios vamos a identificarlas. Una vez presentadas y justificadas, pasaremos a presentar los resultados obtenidos, y los analizaremos para responder a la pregunta que nos planteábamos, para después presentar conclusiones y una propuesta de trabajos de futuros.

\section{Contexto: Foro "Diario de clase" de la asignatura}

La asignatura se cursa en el Máster Interuniversitario Andaluz de Educación Ambiental, un programa de postgrado semipresencial (online-onsite) que se retransmite simultáneamente en siete universidades andaluzas a través del sistema de teledocencia en directo (Adobe ${ }^{\circledR}$ ConnectTM) y con una plataforma online (Campus Virtual por Moodle). Esta simultaneidad evita la duplicidad de clases y la movilidad inter-sede con el consiguiente ahorro económico y ecológico, y el carácter interuniversitario proporciona riqueza de recursos humanos a disposición de cada una de las sedes.

El estudio se ha realizado en el curso 15-16, en el que se matricularon un total de 61 alumnos, distribuidos de la siguiente forma: 10 alumnos en cuatro sedes, 6 en la quinta y 15 estudiantes en la sexta sede.

Este máster ya es objeto de evaluación e investigación en búsqueda de su optimización mediante una evaluación, tanto de las opiniones del profesorado y estudiantes participantes sobre el mismo a través de un análisis DAFO (Debilidades, Amenazas, Fortalezas, Oportunidades, Romero-Gutiérrez et al., 2016; Romero-Gutiérrez et al., 2015), desde las actividades que se plantean en cada una de sus asignaturas (Morales Sánchez et al., 2015), así como desde las competencias que en él se desarrollan (Ponce Morales y Tójar Hurtado, 2014).

En el presente trabajo contribuimos a esta evaluación del máster, focalizando nuestro análisis en una de las asignaturas que en él se incluyen, llamada Enfoque de enseñanza por indagación para educadores ambientales. Esta asignatura pertenece al módulo inicial del máster, común a los dos itinerarios (investigador y profesional) con carácter obligatorio y su contenido está repartido en siete sesiones presenciales de cuatro horas

Análisis del foro online de un máster interuniversitario por aula remota en clave de emociones. Miguel Romero Gutiérrez, María Martínez Chico y M. Rut Jiménez Liso. 
cada una, a las que se suman el trabajo no presencial realizado por los estudiantes, hasta completar las 100 horas de trabajo totales asignadas.

No existe un modelo universal para la integración de la Educación Ambiental en el proceso educativo (López-Alcarria et al., 2014; Perales-Palacios, Burgos-Peredo, \& Gutiérrez-Pérez, 2014) pero sí que podemos afirmar que dicha integración deberá ser implementada en función a diferentes elementos (edad, conocimiento medioambiental, objetivos...) entre los que son fundamentales las creencias y actitudes que tanto docentes como discentes tienen respecto al medio ambiente (Poza Vilches et al., 2016). Por ello es necesario aclarar que la finalidad de la asignatura objeto de estudio es que los estudiantes reflexionen sobre lo que han de saber y saber hacer los futuros educadores ambientales, haciéndoles vivir, de manera práctica, diferentes experiencias de enseñanza-aprendizaje basadas en la indagación (Martínez-Chico et al., 2014) que favorezcan la construcción de un modelo metodológico útil en su futuro profesional. Así, con el objetivo de que los futuros educadores ambientales sean capaces de adaptar, consumir o prescindir de los abundantes recursos didácticos que pueden encontrar en distintas fuentes, la asignatura se centra en el enfoque de enseñanza por indagación como modelo que aporte unos criterios para su selección y/o adaptación. Durante toda la asignatura se incluyen momentos de autorregulación de aprendizajes en el foro "Diario de clase" (fig. 1), y de emociones en la sesión final de la asignatura. En esta sesión final dedicada a la evaluación (y autorregulación de aprendizajes globales), el alumnado consensuó el peso que debía concederse a las tareas realizadas, decidiendo que la participación en el "diario de clase" supusiera un 10\% de la nota total. Esta información es importante pues el alumnado fue participando en el foro conscientes de que sería objeto de calificación y que el porcentaje de esa calificación la propondrían entre todos. A lo largo del curso se generaron un total de 10 hilos o conversaciones en el foro "diario de clase" con un total de 603 mensajes presentados por 61 alumnos. Cinco hilos fueron generados tras cada sesión semanal, cuatro de envío de tareas surgidas durante la sesión que debían ser comentadas y una que surgió de manera espontánea por los estudiantes tras visualizar un documental (actividad 11) al finalizar la asignatura (tabla 1).

\begin{tabular}{|c|c|c|c|}
\hline ACTIVIDAD & DESCRIPCION & $\mathbf{n}$ & CARÁCTER \\
\hline $\begin{array}{l}\text { 1. Ciencia } \\
\text { experimental } \\
\text { indagativa VS ciencia } \\
\text { teórica tradicional } \\
\text { (Sesión 1) }\end{array}$ & $\begin{array}{l}\text { Hilo conversacional posterior a la primera sesión } \\
\text { iniciado por la encargada del día y continuada por el } \\
\text { "discutidor". Este día nos centramos en definir "hablar } \\
\text { ciencia". }\end{array}$ & 43 & Post-sesión 1 \\
\hline $\begin{array}{l}\text { 2. Buenas prácticas I: } \\
\text { Niños/as que hablan } \\
\text { ciencia } \\
\text { (Videos) }\end{array}$ & $\begin{array}{l}\text { Los estudiantes deben buscar videos, imágenes... en } \\
\text { los que aparezcan alumnos/as que "hablen ciencia" y } \\
\text { comentar qué aspectos les hacen "hablar ciencia". }\end{array}$ & 53 & Tarea individual \\
\hline $\begin{array}{l}\text { 3. Sesión Ecourba } \\
\text { (Sesión 2) }\end{array}$ & $\begin{array}{l}\text { Hilo posterior a la sesión sobre la ciudad como recurso } \\
\text { didáctico para la educación ambiental. Las actividades } \\
\text { grupales versaron sobre debate de ortofotos (cambio } \\
\text { de paisaje tras } 40 \text { años de construcción inmobiliaria) y } \\
\text { análisis de ciudades globales y en transición }\end{array}$ & 45 & $\begin{array}{l}\text { Post-sesión } 2 \\
\text { incluye tarea } \\
\text { grupal }\end{array}$ \\
\hline $\begin{array}{l}\text { 4. Ciclo del agua } \\
\text { (Ciclo Agua) }\end{array}$ & $\begin{array}{l}\text { Actividad de dibujo inicial del ciclo del agua y } \\
\text { utilización de los criterios para criticarlo y mejorarlo }\end{array}$ & 104 & $\begin{array}{l}\text { Tarea individual } \\
\text { doble (durante } \\
\text { sesión 3) }\end{array}$ \\
\hline $\begin{array}{l}\text { 5. Hablar, sentir y } \\
\text { pensar ciencia. (Sesión } \\
\text { 3) }\end{array}$ & $\begin{array}{l}\text { Hilo posterior a la tercera sesión donde trabajamos la } \\
\text { necesidad de plantear preguntas, buscar pruebas y } \\
\text { modelizar }\end{array}$ & 42 & Post-sesión 3 \\
\hline $\begin{array}{l}\text { 6. Comentario } \\
\text { artículos Mrs. }\end{array}$ & $\begin{array}{l}\text { Lectura y comentarios del capítulo "globos flotantes, } \\
\text { teorías flotantes" (Dennis, 2000) y la clase de Mrs }\end{array}$ & 63 & Tarea indi \\
\hline
\end{tabular}

Análisis del foro online de un máster interuniversitario por aula remota en clave de emociones. Miguel Romero Gutiérrez, María Martínez Chico y M. Rut Jiménez Liso.

Página 9 de 21 


\begin{tabular}{|c|c|c|}
\hline $\begin{array}{l}\text { Graham y Globos } \\
\text { (Mrs. Graham) }\end{array}$ & Graham (National Research Council [NRC], 2000). & \\
\hline $\begin{array}{l}\text { 7. Proceso de } \\
\text { indagación } \\
\text { (Sesión 4) }\end{array}$ & $\begin{array}{ll}\text { Vivir la secuencia de indagación de "El Garbanzo"1 } & \\
\text { reconocer la necesidad construir y utilizar modelos. } & 48\end{array}$ & Post-sesión 4 \\
\hline $\begin{array}{l}\text { 8. "Pisito": plano de } \\
\text { una vivienda diseñado } \\
\text { por vosotros } \\
\text { (Sesión 5) }\end{array}$ & $\begin{array}{l}\text { Vivir una secuencia de indagación-modelos sobre } \\
\text { eficiencia energética en los edificios. Dos tareas } \\
\text { individuales: subir el plano de una vivienda ideal (pre, } 108 \\
\text { durante sesión 5) y compararlo con la modificación } \\
\text { tras trabajar elementos pasivos que favorecen la } \\
\text { eficiencia energética (post sesión 6) }\end{array}$ & $\begin{array}{l}\text { Post-sesión } 5 \text { y } \\
\text { tareas individuales } \\
\text { de sesiones } 5 \text { y } 6\end{array}$ \\
\hline $\begin{array}{l}\text { 9. Edificios } \\
\text { (Sesión 6) }\end{array}$ & $\begin{array}{l}\text { Hilo posterior a la sexta sesión donde terminamos de } \\
\text { ver elementos pasivos que favorecían la eficiencia } \\
\text { energética de los edificios. Reflexión sobre el proceso } \\
\text { de enseñanza-aprendizaje vivido. }\end{array}$ & Post-sesión 6 \\
\hline $\begin{array}{l}\text { 10. Obsolescencia y } \\
\text { ecodiseño } \\
\text { (Obsolescencia) }\end{array}$ & $\begin{array}{l}\text { Tras recomendar la profesora la visualización del } \\
\text { documental "comprar, tirar, comprar" surge este hilo } 40 \\
\text { sobre la obsolescencia y el ecodiseño. }\end{array}$ & $\begin{array}{l}\text { Espontáneo tras } \\
\text { proponer } \\
\text { documental }\end{array}$ \\
\hline
\end{tabular}

Tabla 1. Listado de hilos o conversaciones y actividades del foro "diario de clase" (tomada de Romero-Gutiérrez et al., s. f., en revisión).

A pesar de existir un total de 61 estudiantes dados de alta entre las diferentes universidades, en el recuento de participantes que publicaron al menos una vez en el foro, tan solo aparecen 53 alumnos. La diferencia coincide con los abandonos del curso realizados a lo largo del año académico.

Cabe destacar las sesiones 4 y 8 puesto que el número de post duplica al del resto de hilos, este hecho ocurre debido a la propia actividad principal de ambas sesiones, en las que los estudiantes debían subir un dibujo previo y posterior a la sesión de docencia. A continuación, presentamos los criterios utilizados y el proceso seguido para analizar las emociones identificadas.

\section{Emociones identificadas en los mensajes de los estudiantes al foro}

Como hemos indicado en el apartado de objetivos, con la finalidad de dar respuesta a la pregunta ¿Qué emociones detectamos en los mensajes de los estudiantes al foro online y en qué momentos de la asignatura?, estas emociones detectadas nos podrán servir de indicador de si nuestra propuesta formativa ha sido o no relevante para transformar sus actitudes y repercutir en su acción futura. Hemos de ser conscientes de que a través del foro podemos obtener información de los momentos críticos o actividades que funcionan como detonantes de las emociones (propias o ajenas) narradas por los estudiantes en sus mensajes al foro. Para un análisis de si ello provocaría un cambio de las prácticas educativas, se requeriría de otros estudios más amplios centrados en su labor como educadores ambientales, algo que no es objeto de estudio en este trabajo.

El procedimiento seguido para la detección de las emociones en las narraciones de los estudiantes lo resumimos en la Figura 2.

\footnotetext{
${ }^{1}$ Puede encontrarse en: http://www.sensociencia.com/sensopildoras/garbanzo

Análisis del foro online de un máster interuniversitario por aula remota en clave de emociones. Miguel Romero Gutiérrez, María Martínez Chico y M. Rut Jiménez Liso. 
Categorización

inductiva de las emociones reconocidas
Comparamos las categorías inductivas

con la categorización apriorística de emociones (Borrachero, 2015)
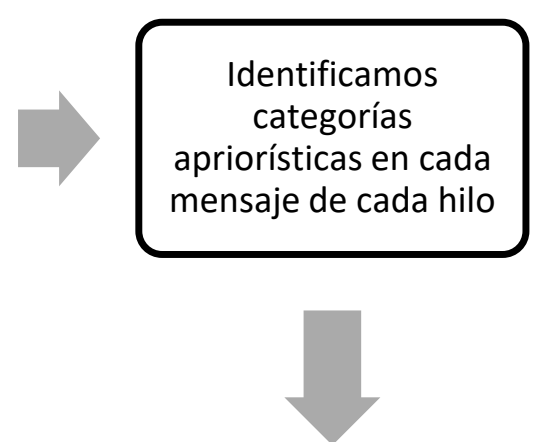

Contabilizamos las categorías declaradas por cada alumno en cada hilo

-0. No reconoce la emoción $\mathrm{X}$

-1. Reconoce la emoción $X$.
Unificamos los mensajes de un mismo alumno en cada hilo

Figura 2. Diagrama de la metodología seguida para el análisis de los mensajes de los foros respecto al reconocimiento de las emociones.

En primer lugar, tres investigadores de manera independiente fuimos identificando frases alusivas a una determinada emoción (categorías inductivas). Al ponerlas en común y analizar el alto grado de consenso (por encima del 90\%), comprobamos la similitud de nuestras categorías emergentes con la categorización de emociones utilizada por Borrachero (2015) ya utilizada por nosotros en un trabajo previo (Martínez-Chico, Jiménez-Liso, López-Gay y Romero-Gutiérrez (2016), primera columna de la tabla 2). Definimos las categorías en función de los indicadores de Mellado Jiménez, Blanco Nieto, Borrachero Cortés y Cárdenas Lizarazo (2013) presentados en la segunda columna de la tabla 2. Tras este consenso definitivo de categorías e indicadores, los tres investigadores procedimos a la relectura y recategorización del foro, llegando a una frecuencia de acuerdo superior al 88\%. En la tercera columna de la tabla 2 exponemos algunos ejemplos característicos de cada categoría.

\begin{tabular}{|c|c|c|}
\hline Categorías & Indicador & Ejemplo \\
\hline ión & $\begin{array}{l}\text { Expresa actitudes positivas hacia/con } \\
\text { algo/alguien. }\end{array}$ & $\begin{array}{l}\text { [...] es un ejemplo a seguir por su entu } \\
\text { amor por su profesión. [A2.UCO5] }\end{array}$ \\
\hline & Expresa una conducta positiva futura. & $\begin{array}{l}\text { También se siente segura } \\
\text { temor a equivocarse. [A11 }\end{array}$ \\
\hline & s negativas hacia/con & $\begin{array}{l}\text { [...] cuando cree que no } p \\
\text { [A11.UMA10] }\end{array}$ \\
\hline & (1) & $\begin{array}{l}\text { [...] porque si ya para mí es un problema, no } \\
\text { querría que fuera algo generalizado. [A3.UCA7] } \\
\text { ¿Realmente sabían lo que era hablar ciencia? } \\
\text { [A4.UAL10] }\end{array}$ \\
\hline intedo & la ausencia de valentía ante un & $\begin{array}{l}\text { [...] no se atreven a improvisar por miedo } \\
\text { fracaso.[A11.UCO9] }\end{array}$ \\
\hline & $\begin{array}{l}\text { Expresa una reacción inmediata de } \\
\text { algo imprevisto o extraño. }\end{array}$ & $\begin{array}{l}\text { mprender que no tiene por qu } \\
\text { absoluta. [A11.UCO4] }\end{array}$ \\
\hline
\end{tabular}

Tabla 2. Listado de Categorías e ítems para evaluar las emociones expresadas.

Análisis del foro online de un máster interuniversitario por aula remota en clave de emociones. Miguel Romero Gutiérrez, María Martínez Chico y M. Rut Jiménez Liso. 
Con los criterios utilizados descritos, vamos a presentar a continuación la metodología utilizada para su análisis, así como los resultados obtenidos.

\section{8. ¿Qué emociones detectamos en los mensajes de los estudiantes al foro online y en qué momentos de la asignatura?}

Para poder realizar un estudio que permita responder a la pregunta del título de esta sección se ha utilizado una metodología descriptiva-interpretativa con datos cualitativos, identificando por un lado qué emociones (propias o ajenas) narran y por otro en qué momentos de la asignatura (hilos del foro) son narradas dichas emociones (propias o ajenas).

\section{1. ¿Qué emociones (propias o ajenas) identificamos en los mensajes de los estudiantes?}

Tras una primera lectura de los mensajes del foro "Diario de clase", unificamos los mensajes de cada estudiante para constatar la frecuencia de presencia de cada categoría por cada estudiante en cada hilo (de manera que si un estudiante explicitaba en el mismo hilo una emoción varias veces, para el estudio esto contará con un valor de 1). En primer lugar, representamos la frecuencia de las emociones detectadas en toda la asignatura, es decir, la frecuencia de estudiantes que expresan una o más veces alguna de las seis emociones (Figura 3).

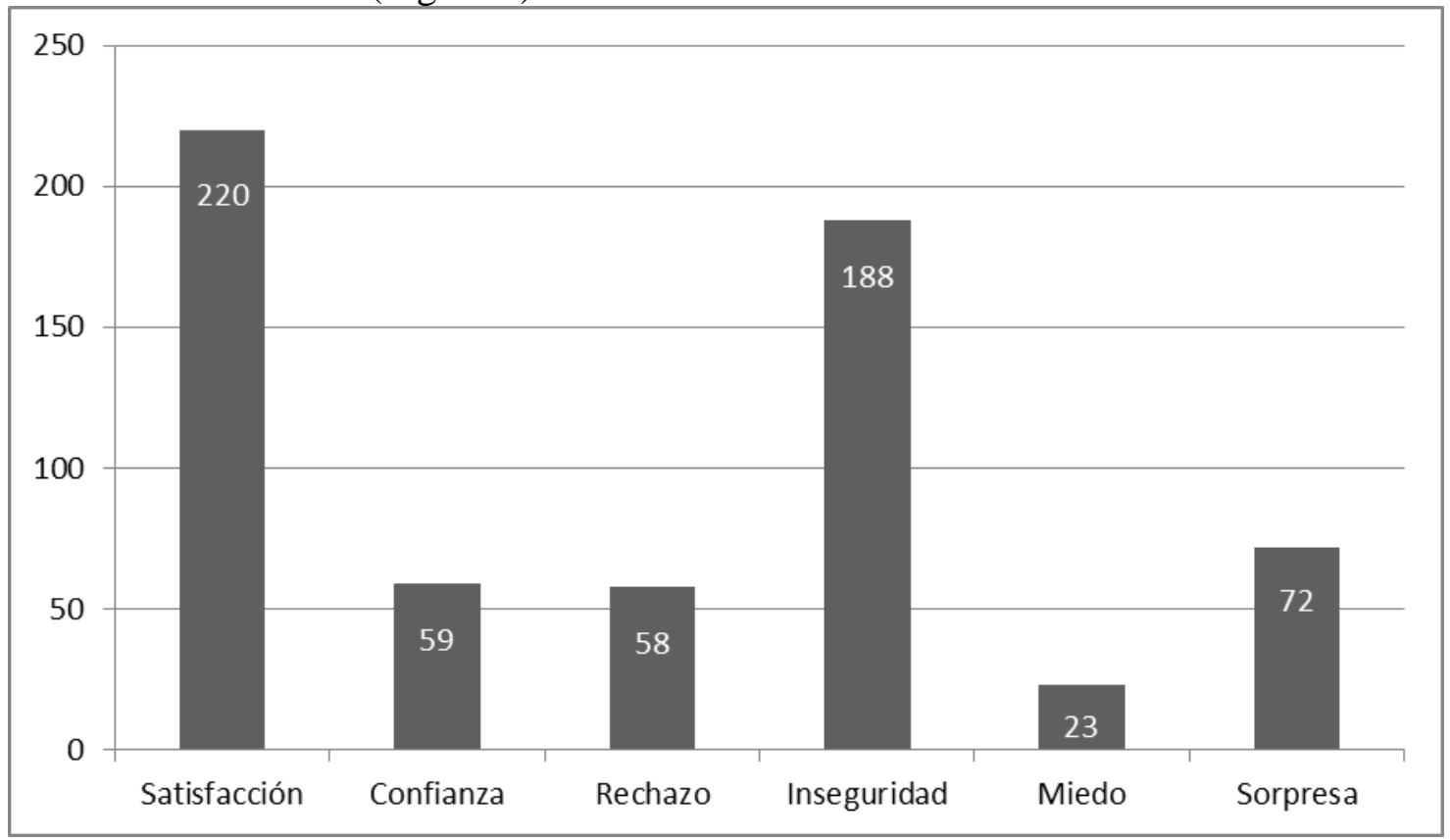

Figura 3. Emociones detectadas en el foro.

Tanto satisfacción como inseguridad obtienen frecuencias muy altas. No queremos clasificar las emociones identificadas como positivas o negativas per se, ni siquiera para el aprendizaje, pues nos parece tan positivo que los estudiantes expresen satisfacción (propia o ajena) durante la asignatura como que destaquen inseguridad (como consecuencia de salir de su zona de confort) pues eso genera cierta insatisfacción con sus concepciones y facilita la construcción del nuevo aprendizaje.

\subsection{Influencia de la sesión-tarea en las emociones detectadas en los diferentes hilos}

Análisis del foro online de un máster interuniversitario por aula remota en clave de emociones. Miguel Romero Gutiérrez, María Martínez Chico y M. Rut Jiménez Liso. 
En segundo lugar, para dar respuesta a en "qué momentos" han expresado esas emociones (propias o ajenas), para analizar la influencia de la sesión-tarea en el proceso emocional, compararemos las frecuencias de acuerdo.

Con el objetivo de no contabilizar de manera repetida una emoción detectada se optó por medir la "frecuencia de acuerdo" entre estudiantes, para ello, una vez identificadas las categorías de cada mensaje, se unificaron los mensajes de cada estudiante en cada hilo, facilitando la generación de la unidad de análisis (en este caso por estudiante en cada hilo) y por tanto midiendo la cantidad de sujetos que identificamos que expresan cada emoción (propia o ajena) por hilo del foro.

Con estos datos se contabilizan, en primer lugar, las emociones identificadas en total por todos los estudiantes en cada hilo a lo largo de las siete sesiones (10 hilos), lo que dará información sobre qué emociones (propias o ajenas) son narradas y en qué sesiones (hilos) las identificamos.

Los datos obtenidos y analizados han sido representados en la Figura 4 de manera acumulativa no porcentual. De esta forma, cada columna describe los hilos analizados y su tamaño retrata la suma de la frecuencia (acuerdo de reconocimiento de emociones propias o ajenas narradas por los estudiantes). A modo de ejemplo, esto significaría, en el caso de la primera emoción "satisfacción", en el Hilo 1, (Sesión 1) ha sido expresada por 12 estudiantes, otros 5 estudiantes han hecho referencia a la "confianza", otros 5 expresan rechazo, 18 Inseguridad y unos últimos 5 estudiantes declaran sorpresa, etc., lo que al sumarlo hace 45 .

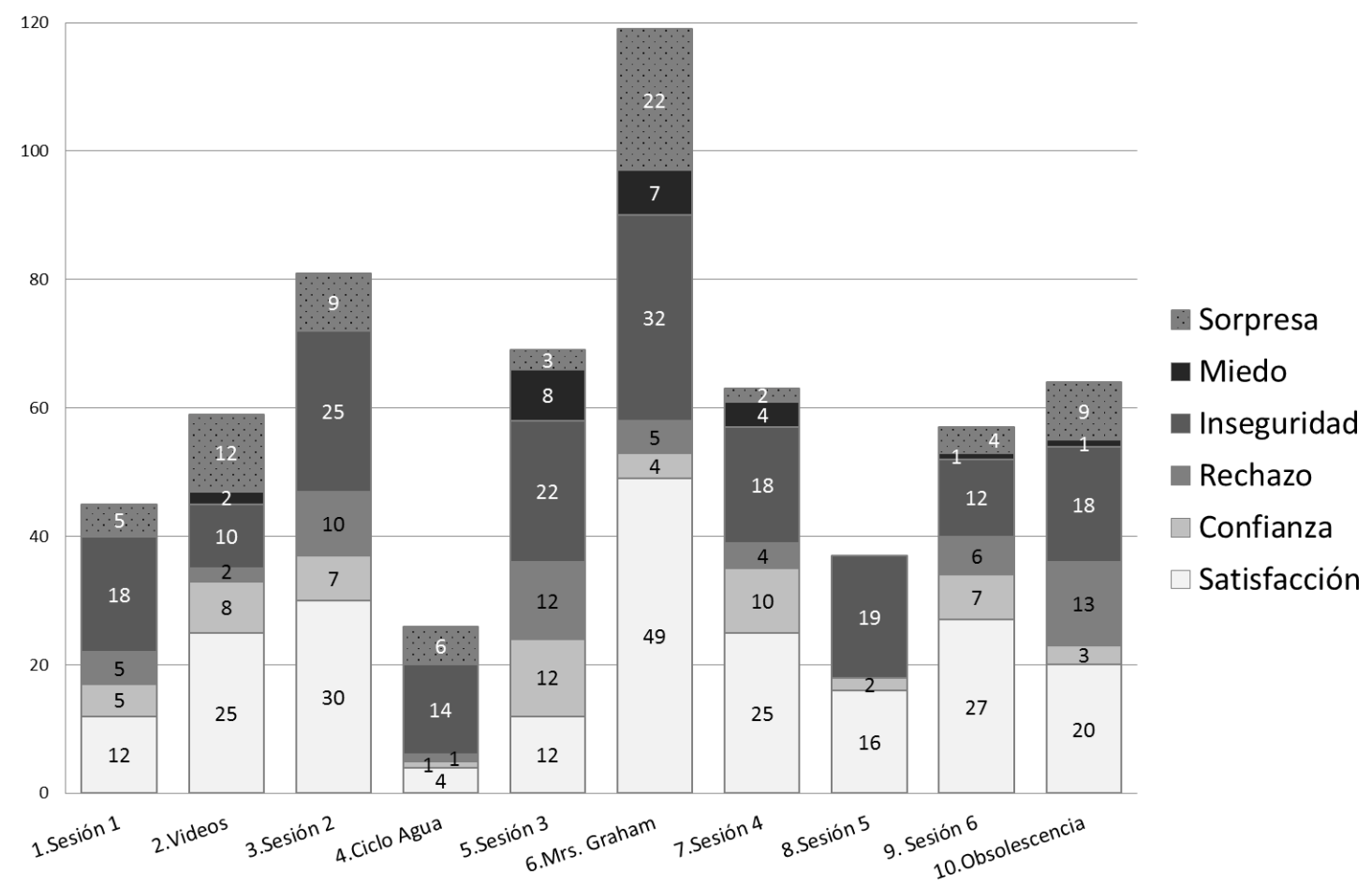

Figura 4. Gráfica acumulativa de respuestas dadas en base a las emociones expresadas.

En la Figura 4 podemos ver que los hilos 6 y 3 son los "más emocionales" en cuanto a la suma total de emociones detectadas, mientras que los hilos "menos emocionales" son los hilos 4 (asociado a la sesión sobre el ciclo del agua) y 8 (asociado a la sesión 5) que fueron los más numerosos en cuanto al número de mensajes, pero en los que el

Análisis del foro online de un máster interuniversitario por aula remota en clave de emociones. Miguel Romero Gutiérrez, María Martínez Chico y M. Rut Jiménez Liso. 
alumnado solo debía enviar sus dibujos previos y posteriores con comentarios de mejora, lo que explica la escasa detección de emociones en ellos.

En todos los hilos hemos detectado satisfacción por parte de los estudiantes (de ahí su frecuencia global tan elevada), incluso en el hilo 6 (asociado a la sesión sobre Mrs. Graham) casi la totalidad de los estudiantes (49 de 53) expresan de alguna manera esta emoción (propia o ajena). De manera similar hemos podido detectar inseguridad en todos los hilos rondando en casi todos ellos una frecuencia de 20 (más de un tercio de los participantes).

Entre las emociones menos detectadas podemos detenernos en "miedo" que no aparece en los hilos 1, 3 y 4 pero sí en los hilos 5 y 6 . Al analizar los comentarios hemos podido comprobar que hacen alusión a falta de seguridad en ellos mismos (miedo que podemos sentir o rechazo a la hora de expresar nuestras ideas [A2.UCA7]) o a la metodología que se propone, más concretamente a la participación activa del alumnado (existiendo como un miedo interno a perder el control de la sesión y ceder la palabra a los niños [A2.UGR8]).

Del mismo modo, nos detenemos, por ser residual, en "rechazo", que aparece con frecuencia superior a 10 en los hilos 3, 5 y 10. En este caso los estudiantes explicitan su rechazo a la actuación del hombre (en los últimos años se han llevado a cabo auténticas barbaridades en entornos naturales [A3.UCA6]), a la metodología cotidiana en las escuelas (cuando había un error, la profesora se dedicaba a gritar y culpar al alumno que se había equivocado[A2.UAL14]) y a la propia actuación con los aparatos rotos (la sociedad de hoy en día no nos permite dedicarle tanto tiempo a arreglar un aparato[A6.UGR4]) y la propia obsolescencia (si no empezamos a cambiarlo desde ya, siendo pesimista, creo que en un futuro cercano no habrá solución[A6. UAL14]).

Esta lectura emocional del foro "diario de clase" nos permite poner de manifiesto que la asignatura no ha dejado impasible a ninguno de los participantes, sino que en sus narraciones han expresado emociones (propias o ajenas) de manera espontánea entrelazadas con la puesta en común de los contenidos conceptuales-procedimentales objetivo de la asignatura.

\section{Conclusiones}

Hemos utilizado el foro online "diario de clase" de una de las asignaturas del máster interuniversitario de Educación Ambiental, además de como actividad docente, como un medio para que el alumnado reflexione y realice un seguimiento de la asignatura, aunando las respuestas de alumnado de siete sedes distantes entre sí y convirtiendo en objeto de investigación lo sentido por el alumnado y su implicación emocional.

La lectura emocional de los mensajes pone de manifiesto que la asignatura les ha producido una implicación emocional. La satisfacción que continuamente manifiestan junto con la inseguridad que les produce denotan que no les ha dejado indiferentes. El hilo conversacional asociando a la sesión en la que se trabajó sobre las experiencias docentes de Mrs Graham y Mrs Duckworth es en el que han expresado más veces emociones (propias o ajenas), junto con la sesión desarrollada por Ecourba, en la que plantearon actividades dos egresados del máster que narraron su experiencia de autoempleo. En ambos casos, se trata de experiencias profesionales concretas que les permiten acercarse a situaciones reales que les resultan relevantes como futuros educadores.

Los resultados obtenidos en términos de generación de emociones con la particularidad del enfoque de enseñanza adoptado en la asignatura (por indagación) confirma el

Análisis del foro online de un máster interuniversitario por aula remota en clave de emociones. Miguel Romero Gutiérrez, María Martínez Chico y M. Rut Jiménez Liso. 
indivisible vínculo entre el aspecto afectivo y la realización de actividades de indagación, como señala Izquierdo Aymerich (2013):

"hacer ciencia es vivir, emociona; aprenderse una ciencia que sólo afirma "verdades" que deben aceptarse por sus aplicaciones y que se suponen objetivas sólo fatiga y aburre."

En el caso particular de la educación ambiental, esta dicotomía emoción-hacer ciencia alcanza una especial relevancia, ya que lo deseable es alcanzar la implicación emocional que conecte el conocimiento racional y emocional, y con ello relacionar el mundo real con nuestra propia identificación y vinculación con la Tierra (Chawla, 2006), generando, de manera profunda, el germen de una nueva cultura a través del cual conseguir transformaciones profundas sobre "saber, hacer, sentir, tener y estar".

Por tanto, al afirmar que los estudiantes narran diferentes emociones (propias o ajenas), coherentes con la metodología usada, con los objetivos de la Educación Ambiental y con el proceder del aula, podemos afirmar que esta asignatura ha contribuido a ser una experiencia relevante de esa visión transformadora de la Educación Ambiental (Sauvé, 2010).

En futuros trabajos ampliaremos este estudio, analizando si la vivencia de esta asignatura ha contribuido a transformar su práctica de Educación Ambiental, analizando si ellos-as contribuyen a que los participantes en sus actividades hablen, hagan, piensen y sientan.

\section{Agradecimientos}

A los estudiantes del Máster Interuniversitario de Educación Ambiental del curso 15-16 por su compromiso con esta actividad docente e implicación en la asignatura. A JuanFer por embarcarse en la aventura de "destripar" los comentarios de sus compañeros-as y suyo propios.

Presentación del artículo: 6 de marzo de 2017

Fecha de aprobación: 15 de septiembre de 2018

Fecha de publicación: 31 de octubre de 2018

Romero-Gutiérrez, M., Martínez-Chico, M. y Jiménez-Liso, M.R. (2018). Análisis del foro online de un máster interuniversitario por aula remota en clave de emociones. RED. Revista de Educación a Distancia, 58. Consultado el (dd/mm/aaaa) en http://www.um.es/ead/red/58/

\section{FINANCIACIÓN}

Este artículo ha sido posible gracias a la cofinanciación del proyecto de investigación Proyecto Sensociencia (P11-SEJ7385) de la Junta de Andalucía y de los proyectos EDU2015-69701-P y EDU2017-82197-P del MINECO.

Análisis del foro online de un máster interuniversitario por aula remota en clave de emociones. Miguel 


\section{Referencias bibliográficas}

Aires, L., Teixeira, A., Azecedo, J., Gaspar, M. I., \& Silva, S. (2006). Alteridad y emociones en las comunidades virtuales de aprendizaje. Teoría de la Educación. Educación y Cultura en la Sociedad de la Información, 7(2), 74-91. Recuperado a partir de http://www.redalyc.org/articulo.oa?id=201017296005.

Álvarez-Pinilla, A. (2014). La medición de la eficiencia y la productividad. Madrid: Ediciones Pirámide.

Area, M., \& Sanabria, A. L. (2014). Opiniones, expectativas y valoraciones del profesorado participante en el Programa Escuela 2.0 en España. Educar, 50(1), $15-39$.

Barberá, E. (2006). Aportaciones de la tecnología a la e-Evaluación. Revista de Educación a Distancia, Número mon, 1-13. Recuperado a partir de http://www.um.es/ead/red/M6/barbera.pdf?origin=publication_detail

Baxter, J. A., \& Haycock, J. (2014). Roles and student identities in online large course forums: Implications for practice. International Review of Research in Open and Distance Learning, 15(1), 20-40. http://doi.org/10.1080/09650799800200051.

Borrachero Cortés, A. B. (2015). Las emociones en la enseñanza y el aprendizaje de las ciencias en educación secundaria. Universidad de Extremadura. Recuperado a partir de http://dehesa.unex.es/xmlui/bitstream/handle/10662/3066/TDUEX_2015_Borrac hero_Cortes.pdf?sequence $=4$

Borrachero Cortés, A. B., Bermejo García, M. L., \& Costillo Borrego, E. (2014). Emociones ante las ciencias y sus posibles causas. Estudio realizado a alumnos del grado de magisterio de educación primaria. En Actas do I Congresso Internacional Envolvimento dos Alunos na Escola: Perspetivas da Psicologia e Educação (pp. 975-990). Lisboa: Instituto de Educação da Universidade de Lisboa.

Boza Carreño, Á., \& Conde, S. (2015). Training, attitude, use and impact of Web 2.0 in higher education: Scale validation / Formación, actitud, uso e impacto de la Web 2.0 en educación superior: Validación de una escala. Cultura y Educación, 27(2), 372-406. http://doi.org/10.1080/11356405.2015.1034531

Brígido Mero, M., Bermejo García, M. L., Conde Núñez, M. del C., \& Mellado Jiménez, V. (2010). The emotions in teaching and learning nature sciences and physics/chemistry in pre-service primary teachers. US-China Education Review, 7(12), 25-32.

Carmi, N., Arnon, S., \& Orion, N. (2015). Transforming Environmental Knowledge into Behavior: The Mediating Role of Environmental Emotions. Journal of Environmental Education, 46(3), 183-201. http://doi.org/10.1080/00958964.2015.1028517

Chawla, L. (2006). Research Methods to Investigate Significant Life Experiences: review and recommendations. Reprinted from Environmental Education Research (1998) 4 (4), pp. 383-397. Environmental Education Research, 12(34), 359-374. http://doi.org/10.1080/1350462980040403

Análisis del foro online de un máster interuniversitario por aula remota en clave de emociones. Miguel 
Chawla, L., \& Cushing, D. F. (2007). Education for strategic environmental behavior. Environmental Education Research, 13(4), 437-452. http://doi.org/10.1080/13504620701581539

Ching, Y.-H., \& Hsu, Y.-C. (2011). Design-grounded Assessment: A Framework and a Case Study of Web 2.0 Practices in Higher Education. Australasian Journal of Educational Technology, 27(5), 781-797.

Cobb, E. (1977). The ecology Imagination in childhood. New York: Columbia University Press.

Dávila Acedo, M. A., Borrachero Cortés, A. B., Cañada Cañada, F., Martínez Borreguero, G., \& Sánchez Martín, J. (2015). Evolución de las emociones que experimentan los estudiantes del grado de maestro en educación primaria, en didáctica de la materia y la energía. Eureka sobre Enseñanza y Divulgación de las Ciencias, 12(3), 550-564. http://doi.org/10498/17609

de la Garza Aguilar, J. (2013). La evaluación de programas educativos del nivel superior en México. Avances y perspectivas. Perfiles Educativos, 35(Número Especial), 33-45.

Díez Gutiérrez, E. J. (2012). Modelos socioconstructivistas y colaborativos en el uso de las TIC en la formación inicial del profesorado Socio-constructivist and cooperative models in the use of I.C.T. in initial teacher education. Revista de Educación, 358, 175-196. http://doi.org/10-4438/1988-592X-RE-2010-358-074

Ehuletche, A. M., De Stefano, A., Banno, B., \& González, E. E. (2007). Ajustes Pedagógicos en entornos virtuales: los proceosos colaborativos. En X EDUTEC. Buenos Aires. Recuperado a partir de http://gte2.uib.es/edutec/sites/default/files/congresos/edutec07/aprobedutec07/do cs/148.doc

Feidakis, M., Daradoumis, T., Caballé, S., \& Conesa, J. (2014). Embedding emotion awareness into e-learning environments. International Journal of Emerging Technologies in Learning, 9(7), 39-46. http://doi.org/10.3991/ijet.v9i7.3727

Fuentes Agustí, M., \& Guillamón Ramos, C. (2006). El uso del foro virtual como herramienta para favorecer el aprendizaje autónomo y en grupo del estudiante en titulaciones presenciales adaptadas a las directrices del EEEs. En A. Méndez Vilas, A. E. Solano Martín, J. A. Mesa González, \& J. Mesa Gonzáles (Eds.), Current Developments in Technology-Assisted Education. Vol3 (pp. 1703-1707). Badajoz: FORMATEX.

Goleman, D. (1996). Inteligencia emocional. Barcelona: Kairós.

Gomera Martínez, A. (2008). La conciencia ambiental como herramienta para la educación ambiental: Conclusiones y reflexiones de un estudio en el ámbito universitario. Recuperado a partir de http://www.mapama.gob.es/es/ceneam/articulos-deopinion/2008_11gomera1_tcm7-141797.pdf

Gregori, B., \& Elena. (2006). Aportaciones de la tecnología a la e-Evaluación. RED. Revista de Educación a Distancia, (Monográfico VI), 1-13. Recuperado a partir de http://www.um.es/ead/red/M6.

Gros Salvat, B., \& Silva, J. (2006). El problema del análisis de las discusiones asincrónicas en el aprendizaje colaborativo mediado / The problem of analysing asynchronous discussions in computer supported collaborative learning. RED. Revista de Educación a Distancia, 16, 1-16. Recuperado a partir de http://www.um.es/ead/red/16

Análisis del foro online de un máster interuniversitario por aula remota en clave de emociones. Miguel Romero Gutiérrez, María Martínez Chico y M. Rut Jiménez Liso. 
Gutiérrez-Martín, A., Palacios-Picos, A., \& Torrego-Egido, L. (2010). Tribus digitales en las aulas universitarias. Comunicar, 34, 173-181. http://doi.org/10.3916/C342010-03-17

Hungerford, H. R., \& Volk, T. L. (1990). Changing Learner Behavior through Environmental Education. Journal of Environmental Education. http://doi.org/10.1080/00958964.1990.10753743

Hyatt, D. F. (2005). 'Yes, a very good point!': a critical genre analysis of a corpus of feedback commentaries on Master of Education assignments. Teaching in Higher Education, 10(3), 339-353. http://doi.org/10.1080/13562510500122222

Izquierdo Aymerich, M. (2013). La química ¿emociona? En V. Mellado Jiménez, L. J. Blanco Nieto, A. B. Borrachero, \& J. A. Cárdenas (Eds.), Las Emociones en la Enseñanza y el Aprendizaje de las Ciencias y las Matemáticas (pp. 307-328). Badajoz: DEPROFE.

Jiménez-Liso, M. R., \& López-Gay, R. (2012). A «Class Diary» Forum To Identify Pedagogical Thinking Changes in Preservice Science. En C. Bruguière, A. Tiberghien, \& P. Clément (Eds.), E-Book Proceedings of the ESERA 2011 Conference: Science learning and Citizenhip [12] (pp. 95-99). Lyon, France: European Science Education Research Association. Recuperado a partir de http://www.esera.org/publications/esera-conference-proceedings/sciencelearning-and-citizenship/strand-12/

Lillis, T., \& Turner, J. (2001). Student Writing in Higher Education: Contemporary confusion, traditional concerns. Teaching in Higher Education, 6(1), 57-68. http://doi.org/10.1080/13562510020029608

López Alcarria, A., Gutiérrez Pérez, J., \& Poza Vilches, M. de F. (2014). Preschool Education Professionals as Mediators of Environmental Health Education. Procedia - Social and Behavioral Sciences, (132), 639-646.

Marques, A. R., \& Reis, P. (2017). Producción y difusión de vídeos digitales sobre contaminación ambiental. Estudio de caso: Activismo colectivo basado en la investigación. Revista Eureka sobre Enseñanza y Divulgación de las Ciencias, 14(1), 215-226. http://doi.org/10498/18857

Martínez-Chico, M., Jiménez-Liso, M. R., López-Gay, R., \& Romero-Gutiérrez, M. (2016). Self-regulation of emotions and feelings: Towards a more complete evaluation of pre-service primary teacher training in science education. En 5th New Perspectives in Science Education. Florence, Italy.

Martínez-Chico, M., López-Gay, R., \& Jiménez-Liso, M. R. (2014). Does our initial training program for primary teachers really work? Design, implementation and analysis of a questionnaire. En C. P. Constantinou, N. Papadouris, \& A. Hadjigeorgiou (Eds.), Science Education Research for Evidence-based and Coherence in Learning (Proceeding of the ESERA 2013 Conference) (pp. 156161). Chipre. http://doi.org/978-9963-700-77-6

Martínez-Villar, A., Gutiérrez Pérez, J., \& Perales-Palacios, F. J. (2016). Evaluando la formación virtual en sensibilización ambiental para sectores profesionales/ Assessing virtual training in environmental awareness for professional groups. Educatio Siglo XXI, 34(3), 137-160. http://doi.org/10.6018/j/275981

Mellado Jiménez, V., Blanco Nieto, L. J., Borrachero Cortés, A. B., \& Cárdenas Lizarazo, J. A. (2013). Las emociones en la enseñanza y el aprendizaje de las Ciencias y las Matemáticas Vol. 2. (V. Mellado Jiménez, L. J. Blanco Nieto, A. B. Borrachero Cortés, \& J. A. Cárdenas Lizarazo, Eds.). España: DEPROFE.

Análisis del foro online de un máster interuniversitario por aula remota en clave de emociones. Miguel Romero Gutiérrez, María Martínez Chico y M. Rut Jiménez Liso. 
Mellado Jiménez, V., Borrachero Cortés, A. B., Brígido Mero, M., Melo, L. V., Dávila Acedo, M. A., \& Cañada, F. (2014). Las emociones en la enseñanza de las ciencias. Enseñanza de Las Ciencias, 32(3), 11-36. http://doi.org/10.5565/rev/ensciencias. 1478

Mokoena, S. (2013). Engagement with and Participation in Online Discussion Forums. Turkish Online Journal of Educational Technology - TOJET, 12(2), 97-105. Recuperado a partir de https://acces.bibl.ulaval.ca/login?url=https://search.ebscohost.com/login.aspx?di rect $=$ true $\& \mathrm{db}=$ eric $\& A N=E J 1015527 \&$ lang $=$ fr $\&$ site $=$ ehost-live

Mora Teruel, F. (2013). Neuroeducación. Solo se puede aprender aquello que se ama. España: Alianza Editorial.

Morales Sánchez, M., Jiménez-Liso, M. R., \& Martínez-Chico, M. (2015). Las actividades de las asignaturas del Máster Interuniversitario de Educación Ambiental. Descripción y análisis en relación a las competencias del título (Trabajo Fin de Máster). Universidad de Almería, Almería. Recuperado a partir de

http://cms.ual.es/UAL/estudios/grados/trabajofingrado/curso/MASTER7034?cur so $=2014-15$

National Research Council. (2000). Inquiry and the National Science Education Standards: A guide for teaching and learning. (S. Olson \& S. Loucks-Horsley, Eds.). Washington, DC.: The National Academies Press. http://doi.org/10.17226/9596

National Research Council. (2012). A Framework for K-12 Science Education: Practices, Crosscutting Concepts, and Core Ideas. (Commitee on a Conceptual Framework for New K-12 Science Edcation Standards \& Board on Science Education. Division of Behavioral and Social Sciences and Education, Eds.). Washington, DC: The National Academies Press.

Noriega Borge, M. J. (2012). Utilización del foro virtual en asignaturas de másteres interuniversitarios en el marco del Espacio Europeo de Educación Superior. En F. Guerra López, R. García Ruiz, N. González Fernández, P. Renés Arellano, \& A. Castro Zubizarreta (Eds.), Estilos de aprendizaje: investigaciones y experiencias [V Congreso Mundial de Estilos de Aprendizaje]. Santander, ES: Universidad de Cantabria.

Onrubia, J. (2005). Aprender y enseñar en entornos virtuales: actividad conjunta, ayuda pedagógica y construcción del conocimiento. RED. Revista de Educación a Distancia, (Monográfico II), 1-16. Recuperado a partir de http://www.um.es/ead/red/M2

Organization for Economic Co-operation and Development. (2016). PISA 2015. Resultados Clave. Recuperado a partir de https://www.oecd.org/pisa/pisa-2015results-in-focus-ESP.pdf

Otero, M. R. (2006). Emociones, Sentimientos y Razonamientos en Didáctica de las Ciencias. Revista Electrónica de Investigación en Educación en Ciencias, 1, 2453.

Perales-Palacios, F. J., Burgos-Peredo, Ó., \& Gutiérrez-Pérez, J. (2014). El programa Ecoescuelas. $\quad P e r f i l e s \quad$ Educativos, $\quad 36(145), \quad 98-119$. http://doi.org/10.1016/S0185-2698(14)70640-3

Ponce Morales, I., \& Tójar Hurtado, J. C. (2014). Análisis de competencias y oportunidades de empleo en una enseñanza de posgrado. Propuesta metodológica de evaluación en un máster interuniversitario de educación

Análisis del foro online de un máster interuniversitario por aula remota en clave de emociones. Miguel Romero Gutiérrez, María Martínez Chico y M. Rut Jiménez Liso. 
ambiental. Profesorado. Revista de currículum y formación del profesorado, 18(2), 171-187. Recuperado a partir de http://digibug.ugr.es/bitstream/10481/33517/1/rev182COL2.pdf

Poza Vilches, M. de F., Gutiérrez Pérez, J., \& López Alcarria, A. (2016). Greening Spanish primary schools: Students and teachers attitudes to centres committed to sustainability. 26 SHS Web Conference., 26(ERPA 2015), 1043. http://doi.org/10.1051/ shsconf/20162601043

Punset, E. (2009). Redes \#49: Educar para fabricar ciudadanos. España: SmartPlanet. 13 Diciembre 2009. Recuperado a partir de http://www.youtube.com/watch?v=9Fjhply6DcA

Rebollo Catalán, M. Á., García Pérez, R., Barragán Sánchez, R., Buzón García, O., \& Vega Caro, L. (2008). Las emociones en el aprendizaje online / Emotions in ELearning. Revista Electrónica de Investigación y Evaluación Educativa, 14(1), 1-23. Recuperado a partir de http://www.uv.es/RELIEVE/v14n1/RELIEVEv14n1_2.htm

Reis, P., \& Marques, A. R. (2016). A Investigação e inovação responsáveis em sala de aula. Módulos de ensino a investigação e inovação responsáveis em sala de aula. Lisboa: Instituto de Educação da Universidade de Lisboa.

Romero-Gutiérrez, M., Jiménez-Liso, M. R., \& Martinez-Chico, M. (2016). SWOT analysis to evaluate the programme of a joint online/onsite master's degree in environmental education through the students' perceptions. Evaluation and Program Planning, 54, 41-49. http://doi.org/10.1016/j.evalprogplan.2015.10.001

Romero-Gutiérrez, M., Jiménez-Liso, M. R., \& Martínez-Chico, M. (sin fecha). Enseñanza por indagación en un foro online de Máster interuniversitario de educación ambiental / Inquiry based science education in an environmental education joint Master degree online forum. Profesorado. Revista del curriculum y formación del profesorado (en prensa, aceptado para su publicación).

Romero-Gutiérrez, M., Martínez-Chico, M., \& Jiménez-Liso, M. R. (2015). Evaluación del máster interuniversitario de educación ambiental a través de las percepciones de estudiantes y profesores en un análisis DAFO / Evaluation of the interuniversity master in environmental education in accordance with the students and teacher. Revista Eureka sobre Enseñanza y Divulgación de las Ciencias, 12(2), 347-361. http://doi.org/10498 - 17257

Romero Cuevas, R. M. (2006). Educación ambiental, revolución en la educación. Tepic, México. Recuperado a partir de http://files.centro-demaestroscuautitlan1524.webnode.mx/200000156-31f1032e9a/Ed Amb revolución en la educación. Sesión 6. Interculturalidad.pdf

Rubio, M. J. (2003). Enfoques y modelos de evaluación del e-learning. Revista Electrónica de Investigación y Evaluación Educativa, 9(2), 101-120.

Rubio Serrano, L., Puig Rovira, J. M., Martín García, X., \& Palos Rodríguez, J. (2015). Analizar, repensar y mejorar los proyectos: Una rúbrica para la autoevaluación de experiencias de aprendizaje servicio. Profesorado, Revista de Currículum y Formación del Profesorado, 19(1), 111-126. Recuperado a partir de http://recyt.fecyt.es/index.php/profesorado/article/view/41025

Ryan, A., \& Tilbury, D. (2013). Uncharted waters: voyages for Education for Sustainable Development in the higher education curriculum. Curriculum Journal, 24(2), 272-294. http://doi.org/10.1080/09585176.2013.779287

Salgado Santamaría, C., González Conde, M. J., \& Zamarra López, M. M. (2013). Innovación y aplicación tecnológica en el ámbito de la Educación Superior

Análisis del foro online de un máster interuniversitario por aula remota en clave de emociones. Miguel Romero Gutiérrez, María Martínez Chico y M. Rut Jiménez Liso. 
universitaria. El empleo de los blogs en las universidades españolas. Historia y comunicación social, 18(Número especial), 613-625. http://doi.org/10.5209/rev_HICS.2013.v18.44353

Sarramona i López, J. (2001). Evaluación de programas de educación a distancia. RIED. Revista Iberoamericana de Educación a Distancia, 4(1), 1-24. http://doi.org/10.5944/ried.4.1.1189

Sauvé, L. (2010). Educación científica y educación ambiental: un cruce fecundo. Enseñanza de las Ciencias, 28(1), 5-18. Recuperado a partir de http://ddd.uab.cat/record/57124

Silva, M. (2005). Educación Interactiva. Enseñanza y aprendizaje presencial y on-line. Barcelona: Gedisa.

Tió Torriente, L., Estrada Sentí, V., González Hernández, W., \& Rodríguez Ortega, R. (2011). Instrumento y herramienta informática para guiar, controlar y evaluar las interacciones de los estudiantes en foros virtuales. Educación Médica Superior, 25(2), 59-96.

Zapata-Ros, M. (2011). Evaluación de la calidad en entornos sociales de aprendizaje. RED. Revista de Educación a Distancia, (29), 1-10. Recuperado a partir de http://www.um.es/ead/red/29/zapata.pdf 